\title{
Produção de conhecimento sobre autismo na escola: uma revisão sistemática na base Scielo
}

\author{
Knowledge production on autism in school: \\ a systematic review in the Scielo basis
}

Kelda de Fátima Pereira' ${ }^{1}$, Beatriz Dittrich Schmitt ${ }^{1}$

'Faculdade Porto das Águas, Porto Belo, SC, Brasil.

Recebido em: 31/03/2016 / Aceito em: 15/04/2016

beatriz_bds@hotmail.com

\section{RESUMO}

Objetivo: analisar a produção de artigos científicos sobre autismo nas escolas, disponibilizados na base de dados Scielo. Método: os descritores utilizados foram "autismo" combinado com "escola". Os critérios de inclusão foram: artigos originais em língua portuguesa; artigos completos publicados em periódicos nacionais; pesquisas de campo no ambiente escolar; e, amostra composta por crianças com autismo. Os dados coletados versam sobre: a revista, o ano de publicação, os objetivos, as estratégias metodológicas e os principais resultados. A busca ocorreu em fevereiro de 2016. Resultados: foram encontrados 21 artigos, sendo que seis atenderam aos critérios de inclusão e foram selecionados para análise. As publicações ocorreram entre 2007 a 2015, nas Revistas: Psicopedagogia $(n=02)$, Brasileira de Educação Especial $(n=02)$, Educação e Pesquisa $(n=01)$ e Psicologia: Teoria e Pesquisa $(n=01)$. Os objetivos dos artigos variaram desde avaliação do perfil psicomotor e da competência social entre crianças típicas e atípicas, avaliação da funcionalidade de crianças com autismo, avaliação dos efeitos de programas de intervenção, verificação do preparo de professores de educação inclusiva e descrição do ensino de habilidades matemáticas. Quanto ao delineamento utilizado, há predomínio de pesquisas quantitativas $(n=05)$. As pesquisas se caracterizaram como transversais $(n=03)$ e longitudinais $(n=03)$. Os participantes dos estudos foram indivíduos com autismo e seus pares sem deficiência, crianças sem diagnóstico concluído e professores. Considerações finais: os resultados demonstraram que programas de intervenção são benéficos para os indivíduos com autismo, quanto a psicomotricidade, cognição, linguagem, interações sociais e habilidades matemáticas (adição e subtração). As avaliações indicaram que os indivíduos com autismo têm melhores desempenhos em testes do que muitas vezes a literatura retratava.

Palavras-chave: Autismo; Escola, Revisão.

\section{ABSTRACT}

Objective: to analyze the production of scientific papers about autism in schools available in Scielo database. Method: the descriptors used were "autism" combined with "school". The inclusion criteria were: original papers in Portuguese; complete papers published in national journals; field research in the school setting; and sample of children with autism. Data collected were about: the journal, year of publication, the objectives, the methodological strategies and the main results. The search took place in February 2016. Results: were found 21 papers, six of which met the inclusion criteria and were selected for analysis. Publications occurred from 2007 to 2015 in journals: "Psicopedagogia" ( $n=02)$, "Brasileira de Educação Especial" ( $n=02)$, "Educação e Pesquisa" ( $n=01)$ and "Psicologia: Teoria e Pesquisa" ( $n=01)$. Aim of papers ranged from evaluation of psychomotor profile and social competence between typical and atypical children, evaluation of autism children functionality, assessment of intervention programs effects, check the preparation of inclusive education teachers and description of teaching math skills. On the design used there is a predominance of quantitative research $(n=05)$. The research is characterized as cross $(n=$ 03) and longitudinal ( $n=03$ ) studies. The study participants were individuals with autism and their peers without disabilities, children undiagnosed and teachers. Closing remarks: the results showed intervention pro- 
grams are beneficial for individuals with autism as the psychomotor, cognitive, language, social interactions and math skills (addition and subtraction). The evaluations indicated that individuals with autism have better performances in tests than often portrayed literature.

Keywords: Autism; School; Review.

\section{INTRODUCÃO}

Os Transtornos Invasivos do Desenvolvimento são uma condição que afetam o neurodesenvolvimento do indivíduo e, assim, acometem mecanismos cerebrais de sociabilidade básicos e precoces. ${ }^{1,2}$ Os Transtornos Invasivos do Desenvolvimento incluem condições associadas à deficiência intelectual (síndrome de Rett e transtorno desintegrativo da infância), condições que podem ou não estar associados à deficiência intelectual (autismo e transtornos invasivos do desenvolvimento sem outra especificação) e uma condição comumente associada à inteligência normal (síndrome de Asperger). 2,3

O Transtorno do Espectro de Autismo (TEA) é um dos mais conhecidos Transtornos Invasivos do Desenvolvimento., ${ }^{1,4}$ O TEA provoca prejuízos na interação social, na comunicação e padrões limitados ou estereotipados de comportamentos ou interesses. ${ }^{1,5-9}$ Geralmente aparece de três a cinco primeiros anos de vida e persiste durante toda a vida. ${ }^{8,9}$ Destaca-se que, para cada indivíduo, o quadro clínico se difere e por isso, há variações na forma e nos graus de autismo. ${ }^{8} \mathrm{E}$ o nível de funcionamento intelectual é extremamente variável, que se estende desde profundo comprometimento a habilidades cognitivas superiores. ${ }^{9}$

Em relação ao número de casos de TEA na população, os valores são bastante controversos e oscilam substancialmente entre os estudos. ${ }^{9}$ Até os anos 1990 , a estimativa de 4 a 5 casos por 10.000 pessoas foi amplamente aceita, apesar de existirem relatos de 20 casos por 10.000 crianças. $^{10,11}$ Gomes e Mendes ${ }^{1}$ relataram que, em 2002, a Associação Americana de Psiquiatria considerou a estimativa de 15 casos em cada 10.000 indivíduos, isto é um caso de autismo em aproximadamente 666 pessoas. Atualmente, a Associação Americana de Psiquiatria estima de 2 a 5 casos por 10.000 indivíduos. ${ }^{3}$ Baird et al. ${ }^{11}$ sugeriram 116, 1 casos em cada 10.000 indivíduos. O estudo de Fombonne $^{12}$ forneceu estimativas de prevalência entre 60 a 70 casos para 10.000 indivíduos. As estimativas de prevalência variam entre 30 a 90 casos por 10.000 indivíduos. ${ }^{13-18}$

Com o intuito de se justificar o elevado aumento da prevalência, acredita-se que ao longo dos anos ocorreu alteração dos critérios de diagnóstico, que os pesquisadores utilizaram métodos diferentes, que as variáveis incluídas nos cálculos consideraram dados urbanos e rurais e, além disso, a localização do país e população do estudo também podem ter influenciado nos valores calculados. ${ }^{11,19,20}$

Há predomínio do sexo masculino, sendo aproximadamente a proporção de 2 a 3 homens para 1 muIher, colaborando com os estudos de Frith ${ }^{21}$ e DSM IV. ${ }^{22}$ Apesar do predomínio no sexo masculino, quando se analisa as possíveis etiologias, não se encontram muitas patologias vinculadas especificamente ao cromossomo $\mathrm{X}$, o que justificaria essa diversidade relacionada ao sexo. ${ }^{23}$ Não há uma causa única conhecida para o TEA, mas em sua maioria, é plausível a causa ser vinculada a anormalidades na estrutura ou função cerebral. ${ }^{8}$ Pesquisas mostram diferenças na forma e estrutura dos cérebros de crianças autistas e investigam ligações entre hereditariedade, genética e fatores ambientais. ${ }^{8}$ Também são aceitas hipóteses de que fatores ambientais e genéticos aumentam a propensão para TEA. ${ }^{9}$

No que se refere às políticas públicas, atualmente o Brasil vivencia o momento da inclusão de pessoas com necessidades educacionais especiais nas escolas, incluindo aqueles que possuem TEA. ${ }^{24}$ É inegável que o processo da inclusão é recente e por essa razão, por vezes, pode ser difícil avaliar o quanto está sendo efetivo. ${ }^{25}$ Em contrapartida, sabe-se que o processo da inclusão escolar traz benefícios a todos os envolvidos, sobretudo às crianças com necessidades educacionais especiais ou seus pares. ${ }^{25} \mathrm{~A}$ inclusão escolar de alunos com necessidades educacionais especiais no sistema regular também envolve a parceria entre os professores da Educação Especial e os professores responsáveis pela sala de aula, a família e a escola a fim de maximizarem as oportunidades de aprendizagem destes alunos. ${ }^{24,26}$

Por lei, os alunos com necessidades educacionais especiais têm assegurado o direito a matrícula em escolas regulares brasileiras desde a Constituição de 1988. ${ }^{27}$ E, em 1996, a Lei de Diretrizes e Bases da Educação Nacional (Lei no. 9.394) ${ }^{28}$ reafirmou a obrigatoriedade do atendimento educacional especializado e gratuito, preferencialmente na rede regular de ensino. ${ }^{28}$ Desde então, cada vez mais as práticas educacionais inclusivas ganharam força no país. ${ }^{2}$

Há evidências que sugerem que muitos estudos com crianças com necessidades educacionais especiais têm sido publicados, mas poucos envolvendo especificamente alunos com autismo. ${ }^{2}$ Para Gomes e Mendes, ${ }^{2}$ é comum que as pesquisas sobre autismo tratem de metodologias utilizadas em estudos de casos.

Com base nessas considerações, o objetivo dessa pesquisa foi analisar a produção de artigos científicos sobre autismo na escola. Para tanto, considerar-se-á a revista e o ano de publicação, os objetivos, as estratégias metodológicas e os principais resultados dos artigos analisados.

\section{MÉTODO}

Essa pesquisa se caracteriza como uma revisão sistemática na base scielo a partir da seleção e da análise de artigos científicos (originais) de periódicos, sobre o seguinte tema: crianças com TEA na escola.

No mês de fevereiro de 2016 foram levantadas e analisadas as publicações relacionadas ao tema de interesse. A busca foi realizada a partir da base de dados Scielo. Os descritores utilizados foram "autismo" combinado com "escola". Utilizou-se o operador lógico AND para a combinação dos termos usados durante a busca das publicações.

Os critérios de inclusão foram: (a) artigos originais em língua portuguesa; (b) artigos completos publicados em periódicos nacionais; (c) pesquisas de campo desenvolvidos no ambiente escolar; e, (d) amostra composta 
total ou parcialmente por crianças com deficiência (TEA). Inicialmente, realizou-se uma leitura dos títulos e resumos dos artigos encontrados na busca. Posteriormente, os artigos selecionados foram obtidos na íntegra e examinados de acordo com os critérios de inclusão estabelecidos.

Para a análise dos estudos, foram considerados os seguintes aspectos: autores, ano e revista de publicação do estudo, objetivos dos artigos, tipo de delineamento, participantes (quantidade e idade) e principais resultados encontrados. Apresenta-se na Figura 1 um organograma dos artigos encontrados e, ato contínuo, analisados.

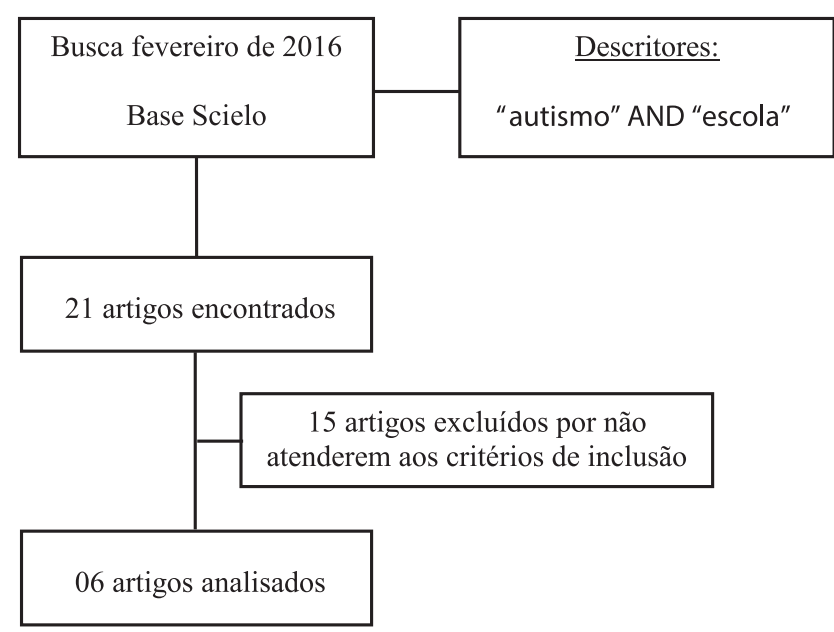

Figura 1 - Organograma dos artigos encontrados.

\section{RESULTADOS E DISCUSSÃO}

A partir da busca, foram encontrados 21 artigos. Destes, 15 foram excluídos porque não atenderam aos critérios de inclusão prestabelecidos. Logo, seis artigos resultaram para análise detalhada (Figura 1).

Acrescenta-se que as razões que motivaram a exclusão dos 15 artigos foram variadas, sendo: seis artigos não eram pesquisas de campo, três artigos foram publicados em idioma inglês ou espanhol, três artigos não continham amostra composta por crianças com deficiência (TEA), dois artigos não foram desenvolvidos no contexto escolar, um artigo foi publicado em periódico internacional.

Com base nessas considerações, evidencia-se que há carência de estudos realizados com crianças com TEA, no ambiente escolar. Esse fato é preocupante, pois atualmente a discussão sobre a inclusão educacional de crianças com defiência tem assumido grandes proporções em âmbito nacional. E, em contrapartida, as discussões afloradas sobre a inclusão desses alunos não se reflete na produção de conhecimento.

É válido destacar que, no ano de 2004 foi publicado pelo Ministério Público Federal, o documento intitulado "O Acesso de Alunos com Deficiência às Escolas e Classes Comuns da Rede Regular" 29 com a finalidade de disseminar os conceitos e diretrizes mundiais para a inclusão, reafirmando o direito e os benefícios da escolarização de alunos com e sem deficiência nas turmas comuns do ensino regular. ${ }^{24}$

As características dos estudos selecionados para análise detalhada são apresentadas na Tabela 1.

Tabela 1 - Informação sobre os artigos analisado (autores, ano de publicação e revista).

\begin{tabular}{lcl}
\hline Autores & Ano & Revista \\
\hline Sandroni; Ciasca; Rodrigues & 2015 & Rev. Psicopedagogia \\
Gomes; Nunes $^{31}$ & 2014 & Rev. Educação e Pesquisa \\
Miccas; Vital; D'antino $^{32}$ & 2014 & Rev. Psicopedagogia \\
Camargo; Bosa $^{33}$ & 2012 & Rev. Psicologia: Teoria e Pesquisa \\
Farias; Maranhão; Cunha $^{34}$ & 2008 & Rev. Brasileira de Educação Especial \\
Gomes $^{35}$ & 2007 & Rev. Brasileira de Educação Especial \\
\hline Rev. $=$ Revista. & &
\end{tabular}

A partir da Tabela 1, observa-se que os artigos foram publicados no período compreendido entre 2007 a 2015 , sendo que a maior concentração das publicações com essa temática ocorreu em 2014 ( $n=02 ; 33,3 \%$ ). Nos demais anos (2015, 2012, 2008 e 2007) foi publicado um artigo em cada ano. Para melhor ilustrar os anos em que ocorreram as publicações dos artigos, recorre-se a Figura 2.

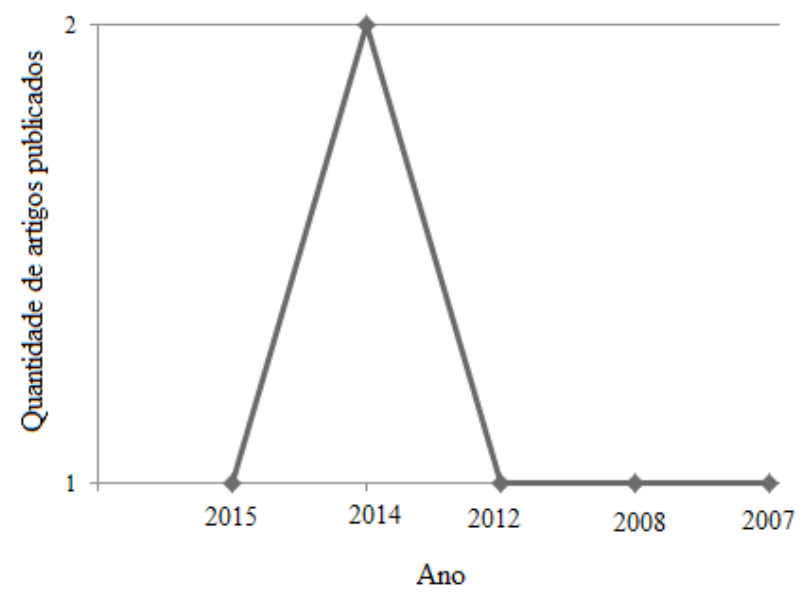

Figura 2 - Ano de pubicação dos artigos selecionados para análise.

No Brasil, mesmo com o aumento de estudos nos últimos anos, foram encontrados poucos autores que abordam sobre esta temática. Ou seja, a temática da inclusão educacional que é tanto evidenciada pelas políticas públicas, no cenário nacional não tem recebido a atenção necessária.

Também é possível notar a partir da Tabela 1, que os artigos foram publicados em revistas distintas, sendo que a Revista Psicopedagogia e Revista Brasileira de Educação Especial concentraram quatro dos seis artigos publicados ( $n=04 ; 66,7 \%$ ). Já, a Revista Educação e Pesquisa e a Revista Psicologia: Teoria e Pesquisa publicaram um artigo cada uma.

Quanto aos objetivos dos artigos, constatou-se que também foram diversificados. No estudo de Sandroni, Ciasca e Rodrigues, ${ }^{30}$ o objetivo foi avaliar e comparar o perfil psicomotor de crianças do ensino infantil com necessidades educativas especiais, antes e após intervenção psicomotora breve.

Em Gomes e Nunes ${ }^{31}$ o objetivo foi avaliar os 
Tabela 2 - Informação sobre aspectos metodológicos dos artigos analisados (delineamento e informações sobre os participantes).

\begin{tabular}{|c|c|c|c|}
\hline \multirow[t]{2}{*}{ Autores } & \multirow[t]{2}{*}{ Delineamento } & \multicolumn{2}{|c|}{ Participantes } \\
\hline & & Número & Idade (anos) \\
\hline \multirow[t]{3}{*}{ Sandroni; Ciasca; Rodrigues ${ }^{30}$} & Quantitativo & 02 crianças com TEA & 5 a 6 \\
\hline & Transversal & 01 criança com DI & \\
\hline & & 02 crianças sem diagnóstico & \\
\hline \multirow[t]{2}{*}{ Gomes; Nunes ${ }^{31}$} & Qualitativo & 01 criança com TEA & 10 \\
\hline & Longitudinal & 01 professora & *Professora: - \\
\hline \multirow[t]{2}{*}{ Miccas; Vital; D'antino ${ }^{32}$} & Quantitativo & 12 indivíduos com TEA & 8 a 22 \\
\hline & Transversal & Professores & *Professora: - \\
\hline \multirow[t]{2}{*}{ Camargo; Bosa ${ }^{33}$} & Quantitativo & 01 criança com autismo & 3 a 4 \\
\hline & Transversal & 01 criança com DT & \\
\hline \multirow[t]{2}{*}{ Farias; Mlaranhão; Cunha ${ }^{34}$} & Quantitativo & 02 crianças com TEA & 3 \\
\hline & Longitudinal & 02 Professoras & ${ }^{*}$ Professora: 35 \\
\hline \multirow[t]{2}{*}{ Gomes $^{35}$} & Quantitativo & 1 criança com TEA & 12 \\
\hline & Longitudinal & & \\
\hline
\end{tabular}

TEA - Transtorno do Espectro Autista; DI - Deficiência Intelectual; DT - Desenvolvimento típico.

efeitos do emprego de um programa de capacitação do professor para a utilização dos recursos da comunicação alternativa e ampliada com um aluno com autismo, por meio de estratégias naturalísticas de ensino. Para Miccas, Vital e D'antino, ${ }^{32}$ o objetivo foi avaliar a funcionalidade de alunos com diagnóstico de transtornos do espectro do autismo.

No artigo de Camargo e Bosa, ${ }^{33}$ os objetivos foram investigar e comparar o perfil de competência social de uma criança pré-escolar com autismo, em situação de inclusão escolar, ao de uma criança com desenvolvimento típico e também investigar a influência do ambiente escolar no perfil da competência social dessas crianças.

Em Farias, Maranhão e Cunha, ${ }^{34}$ o objetivo foi verificar o preparo de professores de educação inclusiva para promoção do desenvolvimento cognitivo de crianças autistas em idade pré-escolar, utilizando a Escala de Avaliação da Experiência de Aprendizagem Mediada. Além disso, foram investigadas concepções acerca da inclusão destes professores, assim como o apoio recebido por eles para realização de sua prática pedagógica no contexto de uma escola considerada inclusiva.

No estudo de Gomes, ${ }^{35}$ o objetivo foi descrever o ensino de habilidades de adição e subtração para uma adolescente com autismo e utilizou procedimentos adaptados com base em descrições sobre o quadro de autismo, princípios de aprendizagem da análise experimental do comportamento, técnicas de ensino e observação direta do repertório da participante. Com base no exposto anteriormente, fica nítido perceber que até o ano de 2016, os artigos que foram analisados demonstram variedade no que tange aos objetivos das pesquisas.

Quanto aos aspectos metodológicos, apresenta-se na Tabela 2 informação sobre o tipo de delineamento das pesquisas e sobre os participantes (quantidade e idade).

As metodologias utilizadas foram distintas, conforme apresentado na Tabela 2. Ocorreu predomínio de pesquisa com delineamento quantitativo ( $\mathrm{n}=05$; $83,3 \%$ ) à pesquisa qualitativa ( $\mathrm{n}=01 ; 16,5 \%)$. Além disso, observa-se que as pesquisas se caracterizaram como transversais ( $\mathrm{n}=03 ; 50 \%$ ) e longitudinais ( $\mathrm{n}$ $=03 ; 50 \%)$. Não obstante, os três artigos com delineamento transversal foram realizados com base em protocolos de avaliação.
É válido destacar que, em virtude de terem sido encontrados e analisados uma quantidade pequena de artigos científicos publicados em idioma português e em periódicos nacionais, há pesquisadores que desenvolveram seus estudos com carácter longitudinal. É sabido que temas e problemas que foram pouco pesquisados cabem a elaboração de pesquisas transversais. No entanto, apesar da quantidade de publicações localizadas ser reduzido, já existe o notável interesse em propor intervenções às crianças com TEA.

No que se refere às informações sobre os participantes dos artigos analisados disponibilizadas na Tabela 2 , nota-se que as amostras compostas por crianças com TEA foram reduzidas e heterogêneas.

Gomes e Mendes ${ }^{2}$ afirmam que é comum as pesquisas sobre autismo tratarem de metodologias utilizadas em estudos de casos, com poucos participantes. Entende-se que o fato de as amostras serem reduzidas não seja uma limitação dos estudos, mas sim um ponto forte. Afinal, reconhece-se a necessidade e a importância de pesquisas que se caracterizam como estudo de caso (individuais ou de grupos) de modo que possam retratar com maestria sobre os casos estudos e sobre a pessoa em sua totalidade e de forma aprofundada, sobretudo quando se trata do TEA - um mistério para a ciência.

Acrescenta-se que no universo acadêmico, ultimamente tem sido constatado que há periódicos (nacionais e internacionais) que, cada vez mais exigem que os artigos submetidos para avaliação utilizem grandes grupos amostrais. E, muitas vezes, há falta de compreensão acerca da complexidade do contexto em torno das deficiências. É fato que pequenos grupos amostrais dificultam a generalização dos resultados. Mas, em contrapartida, para a saúde pública é desejável reduzir cada vez mais os casos de TEA, deficiência visual (cegueira ou baixa visão), deficiência auditiva ou surdez, deficiência física e deficiência intelectual.

$\mathrm{E}$, em parte, consideraram-se as amostras heterogêneas porque incluíram crianças com TEA, sem diagnóstico concluído e seus pares sem deficiência. A faixa etária dos participantes também foi abrangente e por essa razão contribui para a heterogeneidade dos participantes, sendo composta por crianças de três anos até adultos de 22 anos de idade cronológica. Ademais, 
Tabela 3 - Principais resultados dos artigos analisados.

\begin{tabular}{ll}
\hline Autores & Principais resultados \\
\hline Sandroni; Ciasca; Rodrigues $^{30}$ & $\begin{array}{l}\text { Há alteração do perfil psicomotor, habilidades de cognição e linguagem das crianças após as } \\
\text { intervenções. }\end{array}$ \\
\hline Gomes; Nunes & Há mudanças qualitativas e quantitativas nas interações professora-aluno, após as intervenções. \\
\hline Miccas; Vital; D'antino ${ }^{32}$ & $\begin{array}{l}\text { Os alunos com TEA apresentam maiores habilidades nas áreas de percepção sensorial, coorde- } \\
\text { nação motora e tarefas do cotidiano (áreas consideradas de grande comprometimento pela } \\
\text { literatura). Quanto à percepção sensorial e motora as crianças com TEA demonstram hipo ou } \\
\text { hipersensibilidade a diferentes estímulos sensoriais e possíveis dificuldades motoras decorrentes de } \\
\text { desordens voluntárias ou involuntárias. }\end{array}$ \\
\hline Camargo; Bosa ${ }^{33}$ & O perfil de competência social da criança com desenvolvimento típico pouco variou entre \\
& os contextos, mas a criança com TEA demonstrou maior frequência de comportamentos de \\
& cooperação e asserção social e menor frequência de agressão e desorganização no pátio. \\
\hline Farias; Maranhão; Cunha ${ }^{34}$ & Comportamentos que não favorecem a modificabilidade cognitiva estrutural da criança. A \\
& $\begin{array}{l}\text { escola deve ser capaz de qualificar o professor para promover e a inclusão social e educacional } \\
\text { e o desenvolvimento infantil. }\end{array}$ \\
\hline Gomes $^{35}$ & A aprendizagem gradativa das habilidades ensinadas, à medida que a intervenção ocorreu. \\
\hline
\end{tabular}

também se pode considerar que os indivíduos, que atendem aos inúmeros critérios para serem diagnosticados com TEA, apresentam características pessoais e comportamentos diferenciados.

Apresentam-se na Tabela 3 os principais resultados dos artigos analisados.

\section{CONSIDERAÇÕES FINAIS}

Por fim, a partir dos resultados das pesquisas analisadas, pode-se perceber que foram encontrados apenas 21 artigos no período entre 2007 a 2015. Desses, somente seis atenderam aos critérios de inclusão (artigos originais em língua portuguesa; artigos completos publicados em periódicos nacionais; pesquisas de campo desenvolvidos no ambiente escolar; e, amostra composta total ou parcialmente por crianças com TEA). Tendo em vista as dimensões que a inclusão escolar assumiu na atualidade, considerou-se reduzido o número de artigos publicados sobre a temática de autismo nas escolas.

Os estudos analisados foram publicados em revistas distintas e seus objetivos variaram desde avaliação do perfil psicomotor e da competência social entre crianças típicas e atípicas, avaliação da funcionalidade de crianças com autismo, avaliação dos efeitos de programas de intervenção, verificação do preparo de professores de educação inclusiva e descrição do ensino de habilidades matemáticas.

As estratégias metodológicas utilizadas foram, em sua maioria quantitativas, de carácter tanto transversais como longitudinais. Os participantes dos estudos englobaram indivíduos com autismo e seus pares sem deficiência, crianças sem diagnóstico concluído e professores. Os resultados dos artigos analisados demonstraram que o atendimento educacional desenvolvido por meio de programas de intervenção fornecem inúmeros benefícios para os indivíduos com TEA. E os estudos com ênfase em avaliações indicaram que os indivíduos com autismo apresentam melhores desempenhos em testes do que muitas vezes a literatura retratava.

Também é válido ressaltar que cada vez mais as crianças com TEA têm sido diagnosticadas e encami- nhadas para os atendimentos especializados precocemente e isso contribui para seu desenvolvimento global.

\section{REFERÊNCIAS}

1. Klin A. Autismo e síndrome de Asperger: uma visão geral. Rev Bras Psiquiatr 2006;28(1):3-11, DOI: 10.1590/S151644462006000500002

2. Gomes CGS, Mendes EG. Escolarização inclusiva de alunos com autismo na rede municipal de ensino de Belo Horizonte. Rev Bras Educ Espec 2010;16(3):375-96. DOI: 10.1590/ S1413-65382010000300005

3. DSM-IV-TR. Manual Diagnóstico e Estatístico de Transtornos Mentais. Disponível em: <http://www.psiquiatriageral.com. br/dsm4/sub_index.htm >. Acessado em: 17 de fevereiro de 2016.

4. Zuchetto AT, França C, Nasser JP. Adequações, dificuldades e auxílios necessários para o engajamento de um autista em atividade motora. Revista Lecturas, Educación Física y Deportes 2011;16(158).

5. Camargo HPS, Bosa AC. Competência social, inclusão escolar e autismo: um estudo de caso comparativo. Psic Teor e Pesq 2012;28(3):315-24. DOI: 10.1590/S010237722012000300007.

6. Fournier KA, Hass CJ, Naik SK, Lodha N, Cauraugh JH. Motor coordination in autism spectrum disorders: $\mathrm{A}$ synthesis and meta-analysis. J Autism Dev Disord 2010;40(10):1227-40. DOI: 10.1007/s10803-010-0981-3.

7. Pimentel AGL, Fernandes FDM. A perspectiva de professores quanto ao trabalho com crianças com autismo. Audiol Commun Res 2014;19(2):171-8. DOI: 10.1590/S231764312014000200012.

8. AMA. Associação Americana do Autismo. Disponível em: <http://www.autismo.com.br>. Acessado em: 20 de setembro de 2016 às 11 horas e 00 minutos.

9. WHO. World Health Organization. Autism spectrum disorders. Disponível em: < http://www.who.int/ mediacentre/factsheets/autism-spectrum-disorders/en >. Acessado em: 17 de fevereiro de 2016.

10. Wing L, Gould J. Severe impairments of social interaction and associated abnormalities in children: epidemiology and classification. J Autism Dev Disord 1979;9(1):11-29. DOI: 0162-3257/79/0300-0011\$03.00/0 
11. Baird G, Simonoff E, Pickles A, Chandler S, Loucas $T$, Meldrum D, Charman T. Prevalence of disorders of the autism spectrum in a population cohort of children in South Thames: the Special Needs and Autism Project (SNAP). Lancet 2016;368(9531):210-15. DOI: 10.1016/S01406736(06)69041-7.

12. Fombonne E. Epidemiology of pervasive developmental disorders. Pediatr Res 2009;65(6):591-8. DOI: 10.1203/ PDR.0b013e31819e7203.

13. Baird G, Charman T, Baron-Cohen S, Cox A, Swettenham $J$, Wheelwright $S$, Drew A. A screening instrument for autism at 18 months of age: a 6 -year follow-up study. J Am Acad Child Adolesc Psychiatry 2000;39(6):694-702. DOI: 10.1097/00004583-200006000-00007

14. Bertrand J, Mars A, Boyle C, Bove F, Yeargin-Allsopp M, Decoufle P. Prevalence of autism in a United States population: the Brick Township, New Jersey, investigation. Pediatrics 2001;108(5):1155-61. DOI: 10.1542/peds.108.5.1155

15. Chakrabarti S, Fombonne E. Pervasive developmental disorders in preschool children. JAMA 2001;285(24):309399. DOI: 10.1001/jama.285.24.3093

16. Yeargin-Allsopp M, Rice C, Karapurkar T, Doernberg N, Boyle C, Murphy C. Prevalence of autism in a US metropolitan area. JAMA 2003;289(1):49-55. DOI: 10.1001/jama.289.1.49

17. Chakrabarti S, Fombonne E. Pervasive developmental disorders in preschool children: confirmation of high prevalence. Am J Psychiatry 2005;162(6):1133-41. DOI: 10.1176/appi.ajp.162.6.1133

18. Honda $H$, Shimizu $Y$, Rutter M. No effect of MMR withdrawal on the incidence of autism: a total population study. J Child Psychol Psychiatry 2005;46(6):572-79. DOI: 10.1111/j.1469-7610.2005.01425.x

19. Fombonne E. The changing epidemiology of autism. J Appl Res Intellect Disabil 2005;18(4):281-94. DOI: 10.1111/j.1468-3148.2005.00266.x

20. Williams JG, Higgis JPT, Brayne CEG. Systematic review of prevalence studies of autism spectrum disorders. Arch Dis Child 2006;91(1):8-15. DOI: 10.1136/adc.2004.062083

21. FRITH, U. Autism, explaining the enigma. Oxford: Blackwell Pub.; 1989.

22. American Psychiatric Association. Diagnostic and Statistical Manual of Mental Disorders (DSM IV). Washington: APA; 1995.

23. Assumpção JR, Pimentel MCA. Autismo Infantil. Rev Bras Psiquiatr 2000;22(2):37-39. DOI: 10.1590/S151644462000000600010
24. BRASIL. Ministério da Educação. Secretaria de Educação Especial. Política nacional de educação especial na perspectiva da educação inclusiva. 2008. Disponível em: <http://portal.mec.gov.br/seesp/arquivos/pdf/politica.pdf> Acesso em: 17 de fevereiro de 2016.

25. Dota FP, Álvaro DMA. Ensino inclusivo: aspectos relevantes. Revista Psicopedagogia 2009;26(79):124-28.

26. Luiz FMR, Nascimento LC. Inclusão escolar de crianças com síndrome de Down: experiências contadas pelas famílias. Rev Bras Educ Espec 2012;18(1):127-40. DOI: 10.1590/ S1413-65382012000100009.

27. BRASIL. Constituição da República Federativa do Brasil, Brasília, DF, 5 de outubro de 1988.

28. BRASIL. Leis de Diretrizes e Bases da Educação. Lei no.9.394, Ministério da Educação, Brasília, DF, 20 de dezembro de 1996.

29. BRASIL. O Acesso de Alunos com Deficiência às Escolas e Classes Comuns da Rede Regular. Disponível em: < http://pfdc. pgr.mpf.mp.br/atuacao-e-conteudos-de-apoio/publicacoes/ pessoa-com-deficiencia/acesso_alunos _ensino publico_2004 >. Acessado em: 15 de fevereiro de 2016.

30. Sandroni GA, Ciasca SM, Rodrigues SD. Avaliação da evolução do perfil motor de pré-escolares com necessidades educativas especiais após intervenção psicomotora breve. Revista Psicopedagogia 2015;32(97):4-13.

31. Gomes CR, Nunes PRD. Interações comunicativas entre uma professora e um aluno com autismo na escola comum: uma proposta de intervenção. Educ Pesqui 2014;40(1):143-61. DOI: 10.1590/S1517-97022014000100010

32. Miccas C, Vital AAF, D'Antino MEF. Avaliação de funcionalidade em atividades e participação de alunos com transtornos do espectro do autismo. Revista Psicopedagogia 2014;31(94):3-10.

33. Camargo SPH, Bosa CA. Competência social, inclusão escolar e autismo: Um estudo de caso comparativo. Psic Teor e Pesq 2012;28(3):315-24. DOI: 10.1590/S010237722012000300007.

34. Farias $M$, Maranhão AVR, Cunha BCA. Interação professoraluno com autismo no contexto da educação inclusiva: análise do padrão de mediação do professor com base na teoria da Experiência de Aprendizagem Mediada. Rev Bras Educ Espec 2008;14(3):365-84. DOI: 10.1590/S141365382008000300004.

35. Gomes SGC. Autismo e ensino de habilidades acadêmicas: adição e subtração. Rev Bras Educ Espec 2007;13(3):34564. DOI: $10.1590 / S 1413-65382007000300004$.

Como citar: PEREIRA, Kelda de Fátima; SCHMITT, Beatriz Dittrich. Produção de conhecimento sobre autismo na escola: uma revisão sistemática na base Scielo. Cinergis, Santa Cruz do Sul, v. 17, n. 1, mar. 2016. ISSN 2177-4005. Disponível em: <https://online.unisc.br/seer/index.php/cinergis/article/view/7135>. Acesso em: . doi:http://dx.doi.org/10.17058/cinergis. v17i1. 7135 . 